\title{
Integração ensino-serviço na formação em Odontologia: percepções de servidores do Sistema Único de Saúde acerca da prática pedagógica no território
}

\author{
Luisa Sanseverino*; Graciela Fonsêca**; Thiago Silva***; Simone Rennó Junqueira****; Celso \\ Zilbovicius*****
}

\author{
* Bolsista de Iniciação Científica, Faculdade de Odontologia da \\ Universidade de São Paulo \\ ** Professora Adjunta, Curso de Medicina, Universidade \\ Federal da Fronteira Sul (UFFS), campus Chapecó \\ *** Mestre em Formação Interdisciplinar em Saúde pela \\ Faculdade de Odontologia, Universidade de São Paulo \\ **** Professora Associada, Faculdade de Odontologia, \\ Universidade de São Paulo \\ ***** Professor Doutor, Faculdade de Odontologia, Universidade \\ de São Paulo
}

Recebido em 13/12/2016. Aprovado em 23/04/2017.

\begin{abstract}
RESUMO
O presente estudo, de natureza qualitativa, parte do pressuposto de que a inserção dos graduandos do curso de Odontologia em programas de integração ensino-serviço contribui para a formação de profissionais de saúde bucal, bem como melhor adapta o currículo aos requisitos colocados pelas Diretrizes Curriculares Nacionais para os cursos de graduação em Odontologia, quanto à aproximação das necessidades de saúde bucal da população. Nesse âmbito, o projeto buscou analisar a percepção de servidores do Sistema Único de Saúde (SUS) vinculados a uma Supervisão Técnica de Saúde do município de São Paulo, acerca da utilização das Unidades Básicas de Saúde (UBS) como cenário pedagógico de estágios para estudantes de Odontologia. Foram realizadas entrevistas semiestruturadas (gravadas e transcritas) com a Coordenadora de Saúde Bucal, nove cirurgiõesdentistas e dez gerentes das UBS da região. Os resultados, trabalhados pela análise de conteúdo de Bardin, evidenciaram que as percepções são otimistas, revelando capacidade suficiente do serviço para ser utilizado como cenário pedagógico. Apesar do relato de dificuldades associadas à falta de profissionais e de capacitação para preceptoria, as mesmas não se colocam como impedimento para a realização dessas ações. A principal limitação para a realização dessas atividades gira em torno da abertura de canais de comunicação entre as Instituições de Ensino Superior e o serviço. Há necessidade de adaptações político-ideológicas na gestão de integração, visando à sustentabilidade de iniciativas que propiciem uma formação crítica, reflexiva e humanista voltada para o SUS.

Descritores: Educação em Odontologia. Saúde Pública. Sistema Único de Saúde. Serviços de Integração Docente-Assistencial. Pesquisa Qualitativa. Saúde Bucal.
\end{abstract}




\section{INTRODUÇÃO}

O Sistema Único de Saúde (SUS) brasileiro busca uma nova perspectiva de cuidado à saúde no país, de caráter universal, integral e equânime para a população. Suas bases conceituais e práticas, pautadas em fundamentos humanísticos e multidisciplinares, demonstram que a saúde abrange conceitos sociais dentro de uma rede de prestação de serviços de saúde ${ }^{1}$, não se restringindo ao cuidado assistencialista de uma área específica de atuação.

A gestão SUS tem papel importante na formação de recursos humanos da área da saúde, bem como o incremento, na sua área de atuação, do desenvolvimento científico e tecnológico ${ }^{2}$. A formação como política do SUS deve buscar um pensar, da mesma forma em que busca interferir na realidade da população no que diz respeito à sua saúde ${ }^{3}$.

Cabe ao SUS não apenas o dever de atender a população, mas também de garantir que a formação dos profissionais esteja sendo adequada para tal atendimento, garantindo universalidade e equidade no atendimento. $\mathrm{O}$ SUS, bem como as Instituições de Ensino Superior (IES), devem coletar, sistematizar, analisar e interpretar a realidade, afim de guiar uma formação crítica e significativa no que diz respeito à valores e orientações humanísticas e sociais $^{3}$.

Para isso, é fundamental que o sistema de ensino na área da saúde seja articulado com os diversos setores sociais, proporcionando a criação de um conceito de saúde mais positivo, integralizado ${ }^{4}$ e com valores democráticos ${ }^{5}$.

No entanto, notou-se ao longo dos anos que, apesar dessa indispensável articulação ensino-serviço, ainda há barreiras estabelecidas pelas tradições técnico-científicas nas formas de ensino das IES. A carência e negligência de conteúdos humanísticos (sociologia, antropologia, filosofia e psicologia) e preventivos durante a graduação indicam insuficiente formação para atuação de forma completa, que atenda as verdadeiras necessidades da população ${ }^{4,6-8}$.

Essa resistência dos processos de ensino traz consequências diretas no processo de trabalho em saúde no SUS, pois a maioria dos cirurgiões-dentistas que compõem as equipes de saúde ainda continuam classificando a técnica como a característica mais importante para atuação na Estratégia de Saúde da Família $(\mathrm{ESF})^{6}$.

Para contornar esse impasse, ocorreram mudanças envolvendo a saúde e a educação para contribuir com o desenvolvimento de um olhar profissional voltado para a nova política pública. Destaca-se o parecer 776, emitido pelo Conselho Nacional de Educação $(\mathrm{CNE})^{9}$, que instituiu as Diretrizes Curriculares Nacionais $(\mathrm{DCN})^{10}$ específicas para cada curso, as quais objetivam guiar a formação dos profissionais de saúde para focos menos tecnicistas e biologicistas e mais humanísticos. As DCN para os cursos de graduação em Odontologia apontam a necessidade de formação crítica e reflexiva, capaz de atuar de acordo com os princípios multidisciplinares e universais preconizados pelo SUS e valorizando a subjetividade das questões que envolvem o processo saúde-doença.

Apesar das DCN e da ampla revisão de currículos, acredita-se que a grande parte dos cirurgiões-dentistas recém-formados ainda continua atuando com uma prática não integralizada, multidisciplinar e humanística pois, durante a formação, não foram estimulados a refletir de maneira antropológica, histórica, econômica e política $^{11,12}$.

Dentre as possibilidades de medidas que poderiam melhorar o quadro, a realização de estágios envolvendo o SUS merece destaque. Períodos de estágio contribuem para o aprendizado clínico, a autonomia e a 
compreensão do aluno nas formas de organização e gestão do trabalho na área da saúde ${ }^{12}$.

Silva ${ }^{13}$, ao avaliar os cenários do SUS como espaços pedagógicos, propõe que as competências e habilidades descritas nas DCN podem ser aprendidas nos estágios curriculares, contribuindo assim para a formação dos estudantes de Odontologia.

Nesse âmbito, o presente estudo procura analisar as percepções dos cirurgiões-dentistas de Unidades Básicas de Saúde (UBS) do município de São Paulo, bem como de suas respectivas gerências, a respeito da utilização do espaço do trabalho como cenário pedagógico para a realização de estágios curriculares.

\section{METODOLOGIA}

O presente estudo, de caráter qualitativo, foi realizado junto aos atores do serviço envolvidos no processo de integração ensinoserviço da Supervisão Técnica de Saúde do município de São Paulo.

A respectiva região, que contempla uma população de aproximadamente $200 \mathrm{mil}$ habitantes, contém 15 equipamentos de saúde, entre UBS, Pronto-Socorro, ambulatórios de especialidades e Centro de Saúde Escola. Foram selecionadas as 11 UBS que a compõe.

Optou-se por entrevistar o Supervisor Técnico da região e pelo menos o gerente e um cirurgião-dentista de cada uma das UBS, cujo critério para inclusão foi a disponibilidade em participar.

Foram sujeitos participantes das entrevistas onze gestores (identificados com o código ' $G$ '), sendo um Supervisor Técnico de Saúde Bucal e dez gerentes de UBS e nove cirurgiões-dentistas (identificados com o código C). As entrevistas foram realizadas no período de maio a junho de 2015 .

Devido às dificuldades para promoção de encontros entre os participantes para coletar os dados com esse grupo, optou-se por utilizar a técnica de entrevista individual semiestruturada, realizada na UBS de cada sujeito. Constitui-se em uma técnica privilegiada de coleta de informações que oferece a possibilidade de a fala ser reveladora de condições estruturais, de sistemas de valores, normas e símbolos e, ao mesmo tempo, possibilita transmitir, através de um porta-voz, as representações de grupos determinados, em condições históricas, socioeconômicas e culturais específicas ${ }^{14}$.

As entrevistas, que duravam em média 20 minutos, seguiram um roteiro estruturado e abordavam questões relacionadas às necessidades e possibilidades locais e percepções para efetivar-se o processo de transformação do serviço em cenário pedagógico para graduandos em Odontologia.

Para o estudo qualitativo, todos os fenômenos possuem o mesmo grau de importância e preciosidade: a constância, ocasionalidade, a frequência das manifestações, bem como a interrupção, a fala e o silêncio. Interpretar os significados manifestos e desvelar os ocultos são atos indispensáveis para permitir que se descreva, analise e interprete a experiência do sujeito da forma mais aproximada da realidade vivida ${ }^{15}$.

Foi estabelecido, para esta etapa, o uso da análise de conteúdo. Essa técnica foi definida por Bardin ${ }^{16}$ como:

"Um conjunto de técnicas de análise de comunicação visando obter, por procedimentos sistemáticos e objetivos de descrição do conteúdo das mensagens, indicadores (quantitativos ou não) que permitam a inferência de conhecimentos relativos às condições de produção/ recepção destas mensagens".

De acordo com a técnica de $\operatorname{Bardin}^{16}$ são criadas categorias por meio do destaque de 
palavras chaves e/ou constantemente repetidas nos discursos avaliados, bem como a associação de palavras. Para auxiliar nessa etapa, utilizou-se também de uma categorização progressiva ${ }^{17}$, com categorias iniciais, mais específicas, e em seguida, agrupadas em categorias intermediárias, e essas em categorias finais, mais abrangentes, permitindo uma análise detalhada do objeto de estudo.

Para tanto, todas as entrevistas foram gravadas e transcritas, mediante autorização do participante no Termo de Consentimento Livre e Esclarecido. A pesquisa seguiu as normas da Resolução 466/12 e foi aprovada pelo Comitê de Ética em Pesquisa da Faculdade de Odontologia Universidade de São Paulo e da Secretaria Municipal de Saúde de São Paulo (parecer número 1.014.979).

\section{RESULTADOS E DISCUSSÃO}

Foi possível identificar 23 categorias iniciais, as quais foram agrupadas em 6 categorias intermediárias, e estas em 3 categorias finais chamadas de Experiências de integração ensino-serviço já realizadas com alunos de graduação de Odontologia; Potencialidades e limitações da integração ensino-serviço; e Aspectos pedagógicos da integração ensinoserviço.

\section{Experiências de integração ensino-serviço já realizadas com alunos de graduação em Odontologia}

Nessa categoria, foram identificadas falas que relatam a ocorrência ou não de atividades de integração ensino-serviço com alunos de Odontologia e sua frequência.

Cerca de metade dos gerentes e cirurgiõesdentistas entrevistados relataram nunca terem desenvolvido experiências com alunos de graduação. Já entre os que relataram ter realizado algum tipo de atividade com estudantes, a grande maioria declarou a natureza esporádica dessas ações:

“(..) alguns anos atrás veio um grupo."

(G8)

"Essas ações acontecem anualmente na unidade." (G7)

"Eu recebi alunos de Odontologia uma vez". (C9)

Essa insuficiência das atividades no SUS também foi também observada por Finkler et al. (2011), os quais relatam que estudantes somente permaneciam no serviço durante um mês, ao final do curso, objetivando o exercício do aprendizado clínico ${ }^{18}$.

No entanto, é importante ressaltar que, de acordo com os entrevistados, as principais atividades que ocorreram nas UBS foram de conhecimento do território e da própria unidade de saúde:

"Não para ficar diretamente na sala com o pessoal da saúde bucal mas, sim pra conhecer território" (G8)

"Para conhecer meu trabalho, visitar, mas não para efetivamente atuar." (C2)

"Ações coletivas, de conhecimento na comunidade, ações em equipamentos sociais (são feitas)." (G7)

Vale ressaltar ainda que parte dos entrevistados relataram que algumas atividades realizadas foram guiadas pelo Programa de Educação pelo Trabalho para a Saúde (PETSAÚDE) que, apesar de ter se mostrado como uma experiência positiva em outros estudos ${ }^{19,20}$, foi temporária.

"Especialmente na área de Odontologia

[...] houve um estágio até hoje que eles participaram da realização de consultas e procedimentos odontológicos (...) Era um aluno do PET, programa de ensino pelo trabalho." (C9)

Os discursos relataram ausência ou pouca experiência com atividades de integração, o que 
revela que o compromisso do SUS em orientar a formação de profissionais da saúde ${ }^{21}$ ainda parece ser incipiente em termos práticos no que diz respeito à saúde bucal.

Diversificar os cenários de aprendizagem, bem como vivenciar os sujeitos e políticas que participam do processo de cuidado da saúde na prática cotidiana, apresentam-se como questões chave para formação humanizada voltada para o SUS $^{22}$. No entanto, a ocasionalidade das atividades existentes, descrita pelos entrevistados, indica a necessidade de aumentar a aproximação entre o ensino e o serviço, para inserir os alunos na prática do mesmo.

Os discursos vão ao encontro da ideia de que uma reorganização dos processos formativos é fundamental para melhorar a articulação entre o sistema de saúde e as instituições formadoras, no sentido de valorizar a educação em serviço, transformando o mesmo em cenário pedagógico ${ }^{3}$, uma vez que ainda existe uma falta de aprofundamento em formação para saúde pública nos cursos de graduação de Odontologia. $^{23}$

\section{Potencialidades e limitações da integração ensino-serviço}

Nesta categoria foi avaliada a capacidade física e humana dos serviços para serem potenciais palcos de integração com as IES. Foi observado que a grande maioria das limitações relacionadas ao serviço dizem respeito a Recursos Humanos (RH) insuficientes, uma estrutura física não preparada e problemas de gestão.

Diversas falas relataram a necessidade de aumentar os RH de Odontologia, formado e preparado como "tutor" ou "preceptor", para que fosse possível dar adequada atenção aos alunos:

"Aumentar o quadro de profissionais da Odontologia porque estrutura para Odontologia não falta.” (G5)
“(...) entrar em atividade de promoção de prevenção que é uma coisa que a gente ainda fica muito limitado, porque a gente ainda tem a questão de um RH muito pequeno de Odontologia para a demanda da UBS. (...)" (C8)

"O que faltaria hoje seria um $\mathrm{RH}$ de acompanhamento (...)" (G2)

De acordo com as falas, uma vez que a carência de profissionais como auxiliares e técnicos se coloca como um impedimento do próprio serviço, a inserção de alunos de graduação poderia ser algo favorável no que diz respeito ao aumento do quadro pessoal. Seguindo essa linha de raciocínio, os entrevistados relataram que o trabalho dos alunos poderia incrementar a oferta do serviço, com aumento no número de atendimentos oferecido à população.

"Eu acho que pro profissional agrega a ajuda que esse estagiário traria para a unidade bem como para a população de um modo geral (...)" (G1)

“(...) o aluno ia ajudar a gente (...) a melhorar um pouco o serviço.” (C5)

A gestão do serviço apareceu apenas como fator limitador para a realização dessas ações. Foi citada a ausência de um projeto de estágio oficial e organizado partindo de instâncias superiores como a Coordenadoria e a Secretaria de Saúde, bem como a possibilidade de licitações e supervisões.

"Olha, que eu saiba nem tem um projeto nesse serviço." (C4)

“(...) teria que ver com meus supervisores, né, acima da minha esfera, se isso é permitido, se eu devo aceitar (...) então, eu teria que conversar com os superiores, que é a supervisão e a coordenadoria." (G4)

Quando questionados sobre a capacidade física das UBS para receber os alunos, as respostas foram heterogêneas. Alguns profi- 
ssionais acreditam haver espaço suficiente na UBS para a realização de ações coletivas e clínicas com os graduandos. Outros disseram que o espaço físico era muito pequeno, não sendo capaz de receber os alunos. A heterogeneidade das respostas é consequência direta das diferenças estruturais de cada UBS, sendo umas maiores que outras.

Em diálogo com essa constatação, Trajman et $a l .{ }^{24}$ também mostraram que alguns profissionais relataram dificuldades para a realização dos estágios no SUS devido às limitações na infraestrutura (espaço e recursos inadequados) e aos problemas de recursos humanos, como despreparo e falta de tempo.

É interessante notar que, mesmo nos casos em que foi relatado espaço físico insuficiente das UBS para receber os alunos, o fato não foi colocado como excludente para a realização das ações. De acordo com as falas, seria possível fazer uma organização dos alunos, para que frequentassem as UBS em grupos menores. Outro ponto colocado como possível solução para contornar a falta de espaço físico foi a utilização dos espaços sociais cadastrados nas respectivas UBS, como escolas e creches, para a realização das ações coletivas.

"Agora, se você falar estrutura física, aqui é meio deplorável (...)" (C5)

“(..) a gente não tem espaço físico para receber muitos alunos no mesmo período" (G8)

"Nossa sala de odonto é gigante. Os equipamentos sociais que a gente tem, as escolas, as creches, são superreceptivos(...)" (G2)

"O espaço é (suficiente) (...)" (C8)

"A nossa dificuldade em receber alunos, seja da odonto, ou seja, qualquer outro aluno, é espaço físico interno. Pensando numa atividade extra, campo mesmo, território, perfeitamente poderia acontecer." (G2)

"O único limitador é a questão espaço mas acho que com o alunos da odonto não é o caso. Ele poderia estar no território (...)" (G2)

Os profissionais demonstraram muito otimismo pois acreditam que há espaços para realização das atividades com graduandos. Além disso, as ações de promoção de saúde e de prevenção de doenças, que geralmente são pouco frequentes na formação dos estudantes, podem ser inseridas no rol de atividades a serem desenvolvidas pelos estudantes, necessitando apenas a instituição de projetos que estimulem a existência das mesmas.

Os sujeitos da pesquisa revelaram, grande potencial para a realização de atividades coletivas, educativas e preventivas, as quais são de extrema importância para a Odontologia. Os dentistas modernos têm a responsabilidade não só de atuar na área clínica, mas também em políticas públicas, em projetos com cooperativas, grupos comunitários e de envolvimento com a população, desenvolvendo ações de saúde com abrangência intersetorial ${ }^{11}$.

É interessante notar que em grande parte das falas, tanto dos dentistas quanto dos gerentes, houve preocupação com a inserção aluno no contexto de ações coletivas, bem como o otimismo no que diz respeito a sua inserção junto ao serviço.

“(...) poderia fazer orientação de escovação, mesmo na nossa área de abrangência, de escolas, instituições que atendem aí (...)" (G1)

"Ah, eu acho que esse contato com a população e justamente essa ação coletiva nas escolas para eles verem como que é a realidade né, que é ver fora dos muros da faculdade ne. $\mathrm{Eu}$ acho que é bem importante, principalmente o planejamento que a gente fez quando eles vieram 
aqui, para eles fazer uma atividade coletiva, educativa com as crianças, ensinar escovação, fazer uma escovação supervisioanada, eu acho que isso é bem importante." (C3)

"... Mas o que tem de bom aqui é que tem vários espaços para a gente ir, para as ações coletivas. Escovação, flúor, palestras sobre higiene..." (C6)

A possibilidade de realização das atividades de integração ensino-serviço, principalmente daquelas educativas e preventivas, as quais merecem destaque na área de Atenção Primária, é real. A existência de espaços possíveis para receber estudantes nas UBS, conforme relatado pelos entrevistados, fortalece a ideia de que esse encontro ensinoserviço é possível. Não menos importante que a área assistencial, os dentistas das UBS têm real obrigação com ações de promoção e prevenção, as quais muitas vezes mostram-se falhas durante a formação dos dentistas. Inserir os alunos de graduação nesse cenário da prevenção é uma medida que pode ser prontamente colocada em prática, necessitando apenas a instituição de projetos que estimulem a existência das mesmas.

Nota-se também que, para além de atividades educativas, alguns profissionais mencionaram também a possibilidade de inserção dos alunos no sistema em atividades de assistência clínica, sugerindo atuações em procedimentos clínicos.

“...seria bastante interessante se eles, assim como as outras áreas, eles acompanhassem o trabalho do cirurgiãodentista e técnico né e do auxiliar. Eu acho que um estagio mais próximo mesmo da do cotidiano desses profissionais seria bastante interessante." (G9)

“(...) Dependendo do ano poderia fazer atividades clínicasaqui mesmo. Coisas mais básicas tipo restaurações, profilaxia, raspagem (...)"(C6)

Em diálogo com esse pensamento, Fonsêca $^{25}$ descreveu atividades de integração realizadas com alunos de graduação através do programa PET-Saúde, que envolveram atuação clínica dos estudantes com a preceptoria de cirurgiões-dentistas, relevando diversas potencialidades para a formação.

Da mesma forma, a experiência do estágio curricular realizado por alunos do $9^{\circ}$ semestre da Faculdade de Odontologia da Universidade Federal do Rio Grande do Sul (UFRGS), que também abrange atividades clínicas práticas na atenção primária à saúde, revelou grande significado pedagógico, possibilitando melhor abordagem dos conceitos teóricos, aplicados na prática, o que realça a importância da integração ensino-serviço-comunidade ${ }^{26}$.

Como potencialidade, um fator que merece destaque é o preparo dos cirurgiões-dentistas para atuarem como preceptores nas atividades de integração. Segundo opinião unânime dos profissionais entrevistados, todos os membros de suas respectivas equipes de saúde estão aptos a receber os estudantes.

“(...) Aqui todos os profissionais são engajados com a questão do ensino e da pesquisa. (...)" (G2)

"Eu acredito que sim (sou preparada). $\mathrm{Na}$ questão de tentar estabelecer um vínculo, de tentar planejar juntos alguma coisa. A equipe é bem aberta, eu acredito que isso não vai ser um empecilho (...)"(C8)

Foi identificada também a questão da ausência de uma formação específica para os cirurgiões-dentistas atuarem como preceptores.

"Pois é, supervisão do próprio serviço, os profissionais não tem formação para serem preceptores (...)"(G0)

Apesar de os profissionais entrevistados se autodescreverem como preparados para receber 
os estudantes, a ausência de capacitação para abordagem pedagógica relatada pelos entrevistados pode dificultar a transformação do serviço em cenário favorável para a integração.

\section{Aspectos pedagógicos da integração ensino- serviço}

As falas que voltaram o olhar crítico para a questão do ensino odontológico destacaram a possibilidade de Integração ensino-serviço como uma experiência muito positiva e enriquecedora para diversos aspectos pedagógicos.

"Estar junto, ver o dia a dia da saúde pública, seria muito interessante para as alunos (...). A realidade do serviço público é muito diferente daquilo que vocês veem na faculdade de odontologia" (C1)

De acordo com Noro e Torquato ${ }^{27}$, apesar da importância da atuação odontológica em saúde coletiva no SUS, a grande maioria dos cursos ainda apresenta seu maior foco curricular na área clínica. Em diálogo com esse pensamento, muitos profissionais comentaram sobre a carência de conhecimento dos alunos no que diz respeito ao setor público, havendo, portanto, uma necessidade de aprofundar suas bases teóricas na área, inclusive por meio da articulação ensino-serviço.

O conteúdo teórico apresentado aos alunos em sala de aula, com foco tecnicista $e$ biologicista, em muito difere da lógica do SUS. A possibilidade de atuação com outros profissionais, bem como o conhecimento das necessidades dos usuários e a totalidade do que envolve o cuidado com saúde pública, são fatores que poderiam ser aprofundados ${ }^{28}$.

"(...) É uma necessidade da aproximação do ensino nos serviços e uma formação de políticas públicas que, de fato, favoreça essa formação em serviço. A gente teve umas políticas indutoras, né, com a entrada dos alunos nos serviços, mas sem preparo pedagógico dos profissionais, sem previsão de estrutura física, sem revisão dos planos, né, dos processos de trabalho dos profissionais e alguma atividade sempre fica comprometida, ou a assistência ou o ensino." (G7)

“(...)a gente pensar no perfil de formação dos futuros profissionais, né. Porque dentro do que eu tenho de necessidades, ou ... que profissional é esse que eu tô recebendo? É um profissional que tá de acordo com as necessidades que existe? Perguntar: que profissional que eu formo? Né. Odonto, especificamente, a gente sabe que é aquele profissional bastante o quê? Bastante voltado pro atendimento particular (...)" (G6)

É interessante notar que o olhar descrito nas falas vai ao encontro da ideia de que uma formação ideal para o cirurgião-dentista deve prepará-lo tanto para a esfera privada quanto para a pública, formando-o para atender o paciente e a sociedade em sua totalidade ${ }^{7}$.

A integração ensino-serviço foi mencionada ainda como uma grande oportunidade pedagógica tanto para aumentar os conhecimentos clínicos dos alunos, quanto para colocá-lo em contato com a realidade do serviço público. A vivência extramuros permite que o aluno entenda na prática o funcionamento do serviço, com suas particularidades administrativas, bem como as políticas de saúde bucal e as funções do cirurgião-dentista ${ }^{26}$. A ampliação dos cenários de aprendizagem é de essencial importância para que o aluno seja capaz de melhor desenvolver habilidades de clínica integrada, buscando a implementação de práticas interdisciplinares ${ }^{28}$.

"Estar junto, ver o dia a dia da saúde publica seria muito interessante para os alunos. Saber a realidade, saber o que a 
gente passa no nosso dia a dia.(...) A realidade do serviço público é muito diferente daquilo que vocês veem na faculdade de Odontologia”. (C1)

“(...)E pro aluno com certeza ele vai levar o conhecimento que ele vai conseguir absorver desse profissional que vai auxiliá-lo durante a estadia na unidade(...)" (G1)

A ideia descrita pelos entrevistados da importância de oferecer ao aluno de graduação a possibilidade de uma experiência concreta de vivência na realidade do serviço foi também descrita por Toassi et al. ${ }^{11}$ como decisiva na capacitação do aluno de Odontologia, o que ressalta ainda mais a necessidade de instauração desses programas.

\section{CONSIDERAÇÕES FINAIS}

A partir dos resultados obtidos, percebe-se a viabilidade de integração ensino-serviço. Os espaços físicos, bem como os recursos humanos, mostraram-se suficientes para arcar com as atividades de integração, necessitando poucas adaptações, tais como reorganizar os horários dos expedientes e utilizar os espaços sociais cadastrados nas UBS.

De acordo com as percepções dos profissionais entrevistados, o aspecto que mais merece atenção diz respeito à gestão nas instituições, para que se tomem iniciativas que possibilitem constantes parcerias.

Dentro desse olhar, uma vez que estruturalmente o serviço se mostrou capaz para receber as atividades, cabe à IES propor estratégias que visem ao trabalho articulado no planejamento, execução e reavaliação das atividades realizadas pelos graduandos nas unidades do SUS. A iniciativa para abertura de canais e diálogos para melhor estabelecer essa parceria é ponto chave para a ocorrência dessas ações.
Durante o processo de formação do cirurgião-dentista, vivências no serviço em atividades de integração têm papel essencial. Os principais articuladores no processo de implementação de atividades de ensino-Serviço são as IES e o próprio serviço. Atenta-se, no entanto, ao fato de que a inserção da formação para o SUS carrega cunho cultural e políticosocial importante. Dessa forma, há necessidade de adaptações político-ideológicas na gestão de integração, visando à sustentabilidade de iniciativas que propiciem uma formação voltada para o SUS de forma crítica, reflexiva e humanista.

\section{ABSTRACT \\ Service-learning integration in Dental education: Unified Health System professionals' perception on pedagogical practice at the territory}

This qualitative study assumes that the participation of Dental students in servicelearning activities is critical to improving the professional formation for the public health service, according to the requirements of the National Curricular Guidelines for Dental Undergraduate Courses, to fulfill the population needs. For these activities to happen, it is mandatory that the service provides physical and humanistic resources. This study analyzed Unified Health System servers' perception regarding the basic health units as a pedagogic scenario. Semi-structured interviews (recorded and transcribed) were conducted with the Oral Health Coordinator, nine Dentists and ten Managers of basic health units. The results were evaluated according to Bardin's Analysis of Contents Manual. The results showed positive perceptions, revealing enough service capacity to be a pedagogical scenario. Although human resource problems were cited in the reports, they do not restrict service-learning activities. Rather, these activities greatest impediment was the communication between service and highereducation institutions. Therefore, a political and ideological adaptation is necessary to provide 
educational changes for a better critical, reflexive and humanistic dentistry formation for public health service.

Descriptors: Education, Dental. Public Health. Unified Health System. Teaching Care Integration. Qualitative Research. Oral Health.

\section{REFERÊNCIAS}

1. Bulgarelli AF, Souza KR, Baumgarten A, Souza JM, Rosing CK, Toassi RFC. Formação em saúde com vivência no Sistema Único de Saúde (SUS): percepções de estudantes do curso de Odontologia da Universidade Federal do Rio Grande do Sul (UFRGS), Brasil. Interface Comun Saúde Educ. 2014; 18(49):351-62.

2. Brasil. Constituição de 1988. Constituição da República Federativa do Brasil. Brasília: Senado; 1988.

3. Ceccim RB, Feuerwerker LCM. O quadrilátero da formação para a área da saúde: ensino, gestão, atenção e controle social. Physis. 2004;14(1):41-65.

4. Almeida AB, Alves MC, Leite ICG. Reflexões sobre os desafios da Odontologia no Sistema Único de Saúde. Rev APS. 2010;13(1):126-32.

5. Pinheiro R, Ceccim RB, Mattos RA, Burg R. (org.) Ensino-trabalho-cidadania: novas marcas ao ensinar integralidade no SUS. 2 ed. Rio de Janeiro: IMS/UERJ, CEPESC, ABRASCO, 2006. 156p.

6. Sanchez HF, Drumond MM, Vilaça EL. Adequação de recursos humanos ao PSF: percepção de formandos de dois modelos de formação acadêmica em Odontologia. Ciênc Saúde Coletiva. 2008;13(2): 523-31.

7. Lucietto DA. Percepções dos docentes e reflexões sobre o processo de formação dos estudantes de Odontologia [dissertação]. Rio de Janeiro: Escola Nacional de Saúde Pública Sergio Arouca; 2005.
8. Zanetti CHG. A crise da Odontologia brasileira: as mudanças estruturais do mercado de serviços e o esgotamento do modo de regulação curativo de massa. Anais Universitários. Série Ciências Sociais e Humanas. 1999. 21p.

9. Brasil. Conselho Nacional de Educação. Câmara de Educação Superior. Resolução CNE/CES nº7, de 11 de março de 2003. Institui Referencial para as Diretrizes Curriculares Nacionais dos Cursos de Graduação.

10. Brasil. Ministério da Educação. Conselho Nacional de Educação.Câmara de Educação Superior. Resolução CNE/CES No 3, de 19 de fevereiro de 2002. Institui as Diretrizes Curriculares Nacionais do Curso de Graduação em Odontologia. Brasília, 2002.

11. Aerts D, Abegg C, Cesa K. O papel do cirurgião-dentista no Sistema Único de Saúde. Ciênc Saúde Coletiva. 2004;9(1) :131-8.

12. Toassi RF, Davoglio RS, Lemos VMA. Integração ensino-serviço-comunidade: o estágio na atenção básica da graduação em Odontologia Educ Rev. 2012; 28(4):22342.

13. Silva TA. Cenários de prática no Sistema Único de Saúde e as diretrizes curriculares nacionais para o curso de Odontologia [dissertação]. São Paulo: Universidade de São Paulo; 2015.

14. Minayo MCS. Conceito de avaliação por triangulação de métodos. In: Minayo MCS, Assis SG, Souza ER, editores. Avaliação por triangulação de métodos: abordagem de Programas Sociais. Rio de Janeiro: Fiocruz; 2010. 244p.

15. Minayo MCS. O desafio do conhecimento: pesquisa qualitativa em saúde. 12 ed. São Paulo: Hucitec Editora, 2010. 407 p.

16. Bardin L. Análise de conteúdo. 4a ed. 
Lisboa: Edições 70; 2011. 280 p.

17. Silva AH, Fossá MIT. Análise de Conteúdo: exemplo de aplicação da técnica para análise de dados qualitativos. Qualitas. 2015;1(1):1-14.

18. Finkler M, Caetano JC, Ramos FRG. Integração "ensino-serviço" no processo de mudança na formação profissional em Odontologia. Interface Comun Saúde Educ. 2011;15(39):1053-67.

19. Buffon MCM, Carvalho DS, Daniel E, Junior HV, Pecharki GD, Mariot CA, et al. Contribuição do PET-Saúde para a área de Odontologia da UFPR na consolidação das Diretrizes Curriculares Nacionais e do SUS, nos municípios de Curitiba e ColomboPR. Rev ABENO. 2011;11(1):9-15.

20. Fonsêca GS, Junqueira SR. Programa de educação pelo trabalho para a saúde. 1a ed. Curitiba: Appris; 2014. 246 p.

21. Brasil. Ministério da Saúde. Secretaria de Atenção à Saúde. Departamento de Atenção Básica. Coordenação Nacional de Saúde Bucal. Diretrizes da Política Nacional de Saúde Bucal. Brasília: Ministério da Saúde, 2004.

22. Moysés ST, Moysés SJ, Kriger L, Schmitt EJ. Humanizando a educação em Odontologia - uma prática educativa humanizada na área da Saúde coloca o homem como centro do processo de construção da cidadania. Rev ABENO. 2003; 3(1)58-64.
23. Villalba JP, Madureira PR, Barros NF. Perfil Profissional do cirurgião-dentista para atuação no Sistema Único de Saúde (SUS). Rev Inst Ciênc Saúde. 2009; 27(3):262-8.

24. Trajman A, Assunção N, Venturi $M$, Tobias D, Toschi, W, Brant V. A preceptoria na rede básica da Secretaria Municipal de Saúde do Rio de Janeiro: opinião dos profissionais de saúde. Rev Bras Educ Méd. 2009; 33(1), 24-32.

25. Fonsêca GS. Formação pela experiência: Revelando novas faces e rompendo os disfarces da Odontologia 'in vitro' [tese]. São Paulo: Universidade de São Paulo; 2015.

26. Silva AK. A experiência do Estágio Curricular nos Serviços de Atenção Primária à Saúde na formação do cirurgião-dentista: um estudo cartográfico [dissertação]. Porto Alegre: Universidade Federal do Rio Grande do Sul; 2016.

27. Noro LRA, Torquato SM. Percepção sobre o aprendizado de saúde coletiva e o SUS entre alunos concludentes de curso de Odontologia. Trab Educ Saúde. 2010/2011; 8(3):439-47.

28. Takemoto M, Tomazelli K. A Inserção do ensino odontológico no Sistema Único de Saúde. Tecnológica. 2016; 4(1):1-13

Correspondência para:

Luisa Sanseverino

e-mail: luisasanse@ gmail.com

Rua Agostinho Bezerra, 50/111A

05445-070 Vila Madalena - São Paulo/SP 